\title{
Towards the Convergence between IMS and Social Networks
}

\author{
Ramon Alcarria, Tomas Robles \\ Telematics Department \\ Technical University of Madrid \\ Madrid, Spain \\ \{ralcarria, trobles\}@dit.upm.es
}

\author{
Gonzalo Camarillo \\ NomadicLab \\ Ericsson Research \\ Jorvas, Finland \\ Gonzalo.Camarillo@ericsson.com
}

\begin{abstract}
The need of interaction, communication, and data and affections interchange is speeding up the evolution of the technologies that permit the proliferation of social networks. This paradigm permits people with common interests to feel closer and to interchange content. The present work analyzes the necessity of mobile social networks and proposes an integration between the current social networks and the new generation networks by providing new interfaces to the IMS architecture. Some advantages of this integration will be analyzed. Also, the new communication possibilities and enabler utilization (presence, group management) will be taken into consideration. To finalize, we present an example of integration between Facebook and IMS networks through a RESTful interface.
\end{abstract} REST.

Keywords - IMS, social networks, facebook, convergence,

\section{INTRODUCTION}

A new interaction model is needed in a society in which individuals, companies and cities are related and in which users contribute with his suggestions, interesting information and even his own services to the rest of the community.

In order to resolve the evolution requirements of the current society there are some guidelines that must be followed:

The service ubiquity will be improved by the evolution of the technologies that permit users to reach services immediately, using the mobile extended with environment capabilities.

The study of interface technologies allows the integration of social networks with other kind of networks (such as new generation networks) in order to combine their users and enable the interaction between them. This integration should also achieve the convergence of network capabilities, which makes the integrated network more valuable that each network separately.

It is necessary to develop new technologies that permit people to choose freely and easily which services they want and which are not necessary. In addition, the possibility of generating services by users and the existence of network elements that surround the user and his mobile phone will contribute to the Mobile 2.0 paradigm and the evolution from mass society to network society.
Regarding the aging of the population the new interfaces will provide a more active life for old people and the easy access to social networks will permit them to communicate with relatives or social workers.

Most current approaches toward integrating social networks with mobile devices have missed the opportunity to bind the social context with the rich mobile phone network. Most approaches simply extend the Web interface of the social network to the mobile device; that is, you can view the social network through your mobile phone [1] [2]. Other approaches allow synchronization between the phonebook of the mobile phone and the social network [12], but miss the opportunity to include the advantages of new generation networks such as presence, group management and instant messaging.

In order to explain this idea we must simplify the social network functionality by describing it as a set of users who use some capabilities offered by the network to interact among them and also create and consume their own services. This structure can be observed in Figure 1.

An evolution of the social network toward the incorporation of some mobility features modifies this network structure by adding a simple convergence between the mobile network and the social network.

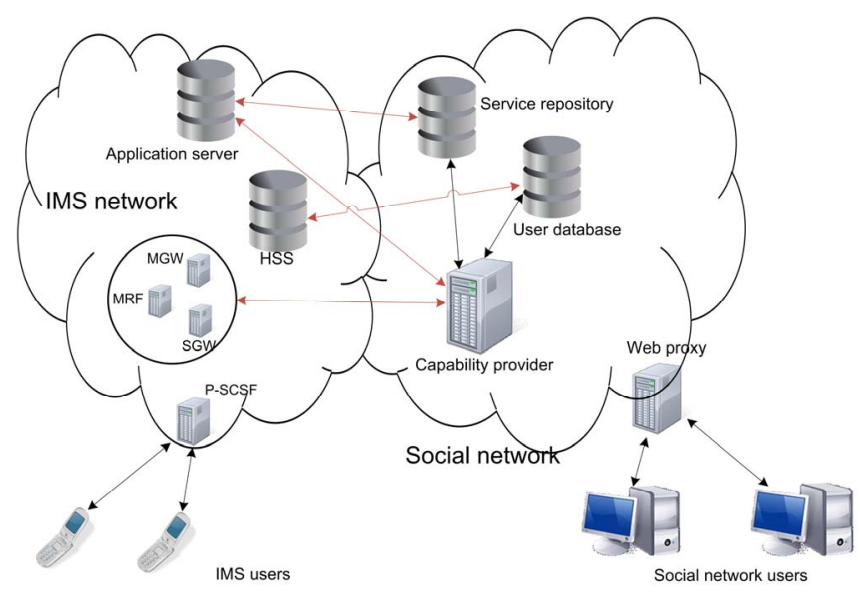

Figure 1. Basic structure of a social network 


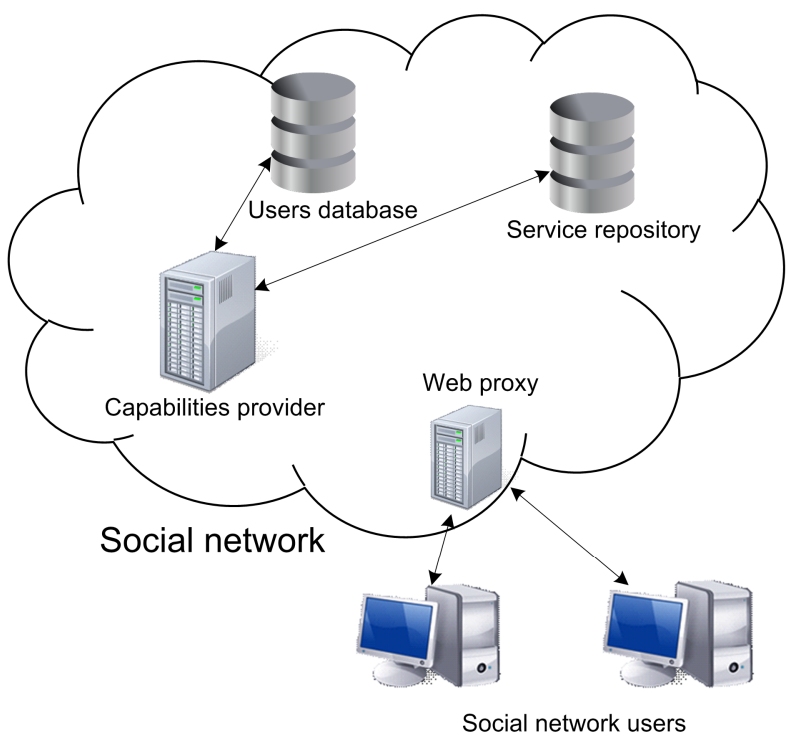

Figure 2. Integration vision

In this case, the number of users is increased but the integrated network offers the same capabilities and services from the original social network.

The network integration presented in this work tries to combine not only the groups of users who use both networks but also the set of services that can be provided and, especially, the interaction possibilities presented in the capabilities that are individually offered by each network. This vision is represented in Figure 2.

As shown, there are some elements in the architecture which provide functions such as resource control (Media Resource control), gateways for multimedia information and signaling (MGW, SGW) and other capabilities that are offered by generic services, defined in the standard, like presence control, list and group management, etc. These elements should interact with the functionalities provided by the social network. The services that are created and published by social network users (stored in the service repository) should be also combined with the services which are deployed into the IMS application server (AS). This task is carried out by deploying typical applications from social networks into the IMS AS.

Finally, the integration between the social network users and the ones registered into the IMS network can be made by deploying applications which access (with appropriate permissions) the user's data in both networks. In this way, a user who belongs to a social network and is also registered in an IMS platform can obtain a list of his acquaintances in both networks and can also know which acquaintances belong to both networks, like him.

The rest of the paper covers the convergence possibilities between social networks and the IMS network and is organized as follows. Section 2 IMS, new interfaces describes new interaction possibilities with IMS through the definition of new interfaces such as an innovative access interface based on Rest. In Section 3 IMS and Facebook integration a proposal of convergence between IMS and social networks is presented. This work has been validated by the implementation of a proof of concept which is also explained. Finally, Section 4 Summary and Conclusions contains the conclusions of this work and some guidelines for a future work in this field.

\section{IMS, NEW INTERFACES}

IMS is now emerging as the key driver for merging the Internet world with the mobile world. The IMS is based on the Session Initiation Protocol (SIP) [3]. The IMS is more than just a protocol, it is an architecture for the convergence of data, service control, mobile networks and is based on a wide range of protocols, most of which have been developed by the IETF [4].

At the same time, new, highly social services have appeared in the Internet world, the so-called Web 2.0 [5] services. The key point behind Web 2.0 services is the change in the philosophy for design and development. The user becomes the centre of the environment and the Internet becomes the platform for developing and delivering services.

Moreover, there is a new tendency to include communication features and other network-related capabilities in these Internet services. Thus, the IMS model fits perfectly with the Web 2.0 services model and a new field for providing new interfaces to the IMS network is opened [6][7].

Network operators are interested in moving from a network-centric approach to a user-centric approach and in becoming platform providers for service construction, provision and consumption. Thus, also from the provider's point of view, the convergence between the network world and the Internet services world becomes an evolution of the utilization of communication networks to gain access to services. This convergence enables the research of how to use the Web 2.0 resources to enrich the services provided by operators.

The IMS access through the Web is achieved by the provision of Application Programming Interfaces (APIs), which turn the IMS offered functionalities into Web services. The IMS applications can be embedded into Web 2.0 services as web-widgets such as the iGoogle Instant Messaging gadget or some Facebook applications which provide voice and presence services. The features that are provided by IMS can be directly used by other applications, invoking different methods of the IMS offered APIs. In this case Web applications must be modified if we want to add some new API invocations but, in a long term, this approach permits better applicability.

We have observed that web services are divided into two different categories:

- Legacy standard web services defined by 3GPP OSA Parlay Parlay-X standards (address list, short 
messaging, audio and third-party call, multimedia conference, presence, location, payment, etc)

- New Web 2.0 and community-oriented web services defined by Google, Yahoo, Orange, BT, Facebook and others (contacts and friends management, social events, personal profile, content, presence and location, conference, etc).

The services within the first group are typically based on the SOAP protocol whereas Web 2.0 services are based on REST. Since the goal of this work is to contribute to the integration of social networks with the IMS architecture we have decided to use a Restful interface.

The IMSInnovation Project [10] opens up the opportunities of the IMS application developers. This project has enabled the development of a Java toolkit, located into a Web portal, which provides some APIs to access to IMS and to use its functionalities.

The API provision is located in a server called Server API, implemented over the IMS architecture, as shown in Figure 3. This server implements a SIP servlet (JSR 289) [9] for the communication to the different IMS architectural elements (SCSF, HSS, Presence Server, etc), gathered together in the IMS Core API module, in Figure 3. Over the IMS Core API module a Communication Service (CoSe) API is added. The functionality of the CoSe Services is to provide APIs for using the standardized services of the IMS.

\section{IMS AND FACEBOOK INTEGRATION}

The Ericsson Research group has created a TestBed [8] whose objective was to ease the development of new thirdparty applications for the IMS by providing web developers

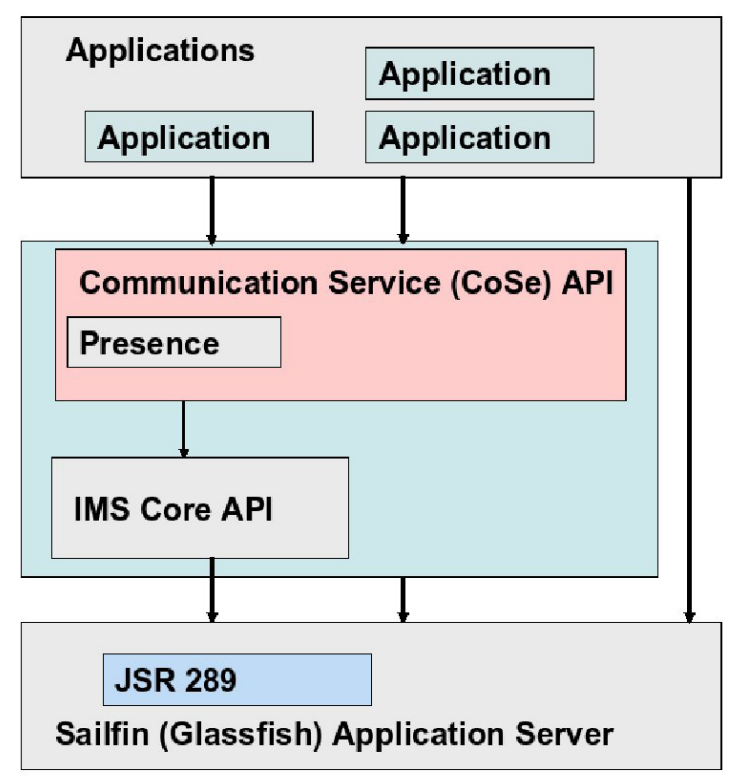

Figure 3. Server API architecture. with Java APIs for Java ME and Java Enterprise Edition. With this TestBed, we have developed a proposal of design and implementation of the integration between the IMS and the Facebook social network. In this section the pursued objective and the procedure to make this proof of concept is explained.

\section{A. Objective}

The objective of the integration between the IMS platform and the Facebook social network is, not only to gain access and interconnection between users who are located into different networks but also to achieve the convergence of functionalities and services which are found in these networks, as seen in Figure 2.

The following IMS functionalities are obtained by using the Restful IMS API, designed in NomadicLab [13] and any Facebook application is able to consume them:

- Registration to the IMS network supported from client terminals.

- Instant messaging can be used inside a session by using the MSRP (Message Session Relay Protocol) protocol. Outside a session, IM is supported by the SIP MESSAGE request.

- $\quad$ File transferring supported by MSRP protocol.

- Presence managing: Users can publish their presence information and receive notifications of a presence change if they have subscribed to other users' presence. Users can also set presence authorization rules so that they can control what presence information will be given to which watchers and under which conditions.

- Buddy list managing, by using the XCAP (XML Configuration Protocol) to access to the XDMS (XML Document Management Server) server.

\section{B. Integration revealed}

Regarding the functionalities that are offered by IMS to the social networks we make possible a communication between enablers so that new options can be presented to both IMS and Facebook users.

Since file transfer is available through the Restful API, images and video interchange is allowed between the IMS and Facebook network. In this way, a user who takes a photo with his mobile SIP user agent can upload this image to his Facebook account in an immediate way or rather share it with his friend network in the IMS system. To make this happen, we must deploy an application that allows the communication with the IMS network and that is also accessible from Facebook and can interact with its servers.

The IMS presence information is also improved since it obtains information about the user status in the Facebook network or when it was the last time the user was online. This information is stored in the presence document of a peer in PIDF (Presence Information Data Format) format and is obtained by other users that are subscribed to this 
peer by the invocation of the getPidfDocument [10] method in the REST API.

Finally, the IMS instant messaging functionality is integrated with the sending of notifications and Facebook private messages. We provide a middleware which checks the required action and chooses the appropriate sending method. This middleware is integrated into the application that is provided by the IMS AS.

The integrating architecture of this work and the interaction process are shown in the following figure:

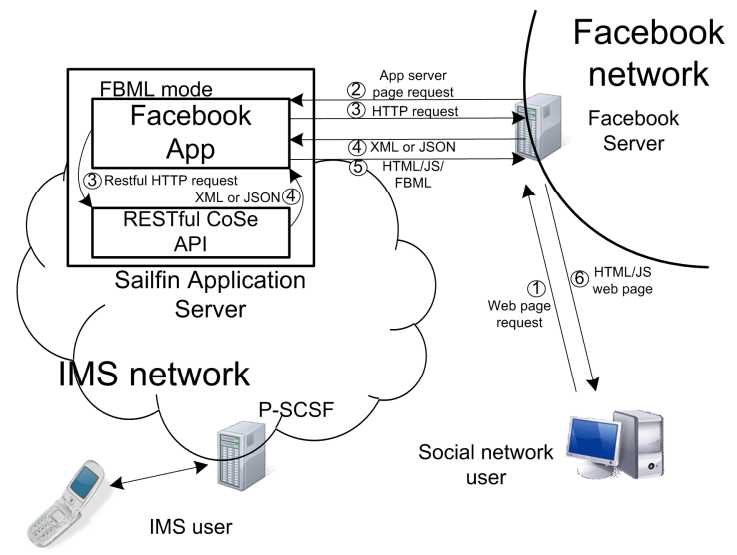

Figure 4. Integration architecture and process.

This integrating architecture reveals that the proof of concept, described in the next section, allows a Facebook user to use the capabilities from the IMS-Facebook integration to access to IMS functionalities while he is taking advantage of the features that are offered by Facebook servers.

As we can see in Figure 4, the Facebook App is a FBML application. When a user requests an application on Facebook (for example, http://apps.facebook.com/someapp), how the Facebook server deals with the request depends upon whether it is an iFrame or FBML application. If it is an iFrame application, the Facebook server returns a Facebook web page with the Facebook chrome that contains an iFrame into which the application will be loaded. If it is a FBML application, instead of having the application be a separate entity living in an iFrame, the application becomes part of the HTML page returned from the initial request to the Facebook server. In this case, the Facebook server proxies every call to the server deploying the Facebook application. In addition to returning HTML and JavaScript, the server-side pages can return FBML, which is turned into HTML and JavaScript by the Facebook server before returning it to the user's browser.

Once the Facebook application has been published in the IMS AS the integration between IMS and Facebook network is enabled. The interaction process of the elements which participate in the architecture is described in Figure 4 and is explained below:
1. The user, which is connected and logged into the Facebook network, makes an HTTP request to the Facebook server to run the Facebook application.

2. The Facebook server sends a request to the Sailfin Application Server. In this case, session info is sent with the request as POST URL variables so the application server page knows that the request is coming from Facebook and which user is making the request.

3. The application server page is executed, making any calls to databases or other servers as needed, including calls to the Facebook server using its APIs. In this proof of concept the used API is the Facebook Java Api [11]. API calls must include authentication info, a signature for the call and an MD5 hash of the session key, the application secret key and other information. At the same time, the application interacts with the APIs that are provided by the CoSe layer to take advantage of the IMS-provided functionalities, explained in Section III.A.

4. The Facebook server returns requested data to the application as XML- or JSON-formatted data. In addition to this, it will be produced an invocation response from the API CoSe methods, with XML or JSON format as well.

5. The application returns a page to the Facebook server containing HTML/JS and additionally FBML tags, used for creating dialog boxes, getting a user's name or redirecting to another page. These FBML tags are replaced by the Facebook server with HTML and JS before returning the web page to the user's browser.

6. The Facebook server returns an HTML/JS page to the user's browser which is displayed.

\section{Proof of concept}

As a validation of this work a Facebook integrated application has been developed. This application combines the access to Facebook functionalities (friend lists, presence and instant notifications sending) with the IMS enabler interaction, described in III.A Section (file transferring, enabler accessing, presence and contact management).

From a functional point of view the application is divided into three parts. The first one contains an authentication mechanism for both Facebook and IMS networks by requesting an id and a password. From this moment the user is authenticated into both networks and the application accesses to the provided functionality so that it can integrate some capabilities from both networks.

The second part consists of a Resful API test. It invokes some API methods regarding to group and friend-list management and presence information provision.

The last part permits the integration between networks by deploying a list with the Facebook and IMS users that have a connection with the logged user. In addition to his name and a main picture the application shows the presence information in these two networks and enables the sending 
of instant messages. If a user is connected to the Facebook network the message is sent as a notification and will appear in the bottom-right corner in the Facebook main screen. However, if the user is connected to the IMS network and the IMS presence server detects this, the message will be transmitted to the specified user agent in the presence document through a SIP MESSAGE reply.

Another question to solve is how to determine if a user with IMS account is also member of the social network in order to combine the presence information of both networks. The solution, in this case, is to include in the IMS presence document of the user, in PIDF format, the user id (uid) used in Facebook. In this way, by obtaining the IMS presence information of a contact it is immediate to identify this user in the Facebook network and, providing that he has the appropriate permissions, to check his presence in the mentioned network.

Figures 5, 6 and 7 show some screenshots of how these three parts work.

\section{SUMMARY AND CONCLUSIONS}

The advantages of the integration between social and mobile networks have been mentioned throughout this paper. The current work in this field is based on providing access to the social networks through the mobile phone, using these devices just as Web browsers. This work tries to go a step further by enabling the integration between new generation networks and social networks by three levels:

- In the user level, by providing some mechanisms that make possible to identify a user in different networks and to enable the interaction with other users.

- In the capability level, by the design and the provision of applications which use APIs for the communication with the IMS network and the social networks.

- In the service level, by using an IMS application server to deploy Facebook applications.

To achieve this integration it is necessary to provide some applications that can access to IMS features as well as interact with social network servers. To make possible that the applications can access to the IMS provided features Ericsson Research has developed an API server, whose architecture is shown in Figure 3, which provides different interfaces for IMS access. By this way the different standardized services of IMS can be used.

As a proof of concept, we have developed an application that tests the main methods of the REST interface for IMS access and allows the integration between Facebook users and IMS users. The integration of capabilities is reflected in this implementation by providing an instant messaging tool, which chooses the transmission channel depending on the presence information of a user. If the user is logged in the Facebook network the message is sent as a notification and, otherwise, if the user is found in the IMS network, using a SIP User Agent, the message is sent to the Agent as a SIP MESSAGE reply.

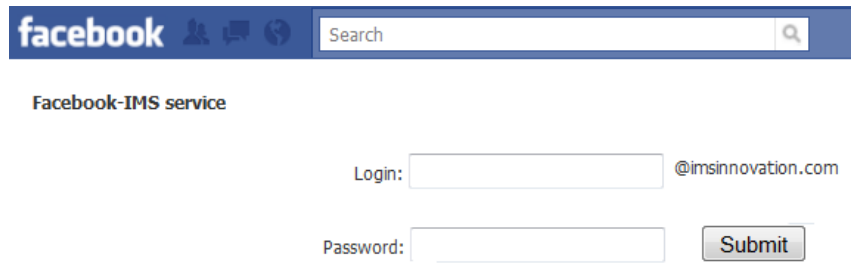

Manage IMS Friends

View IMS \& Facebook Friends

Figure 5. Proof of concept, part one.

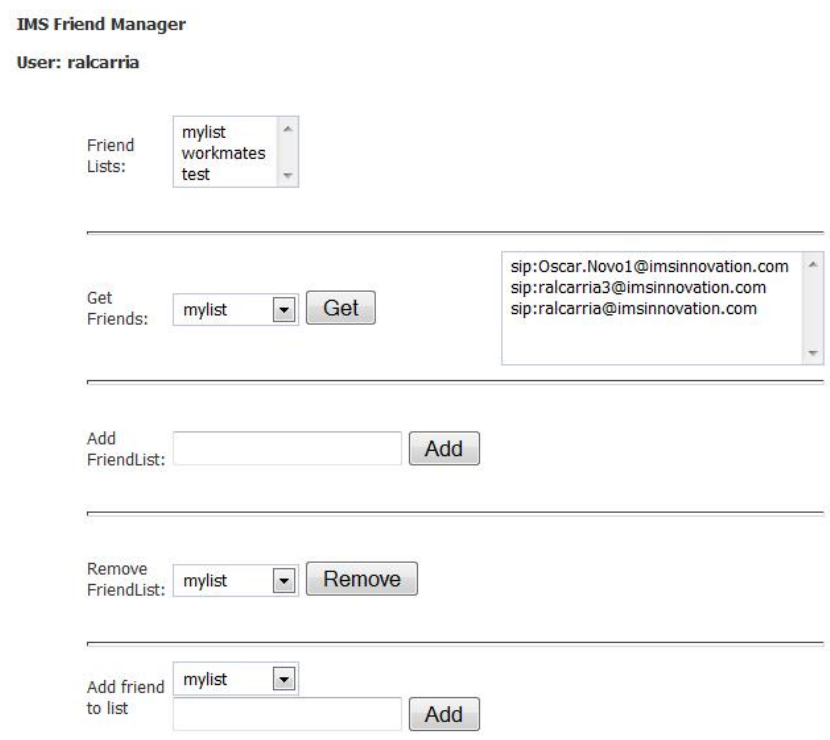

Figure 6. Proof of concept, part two.

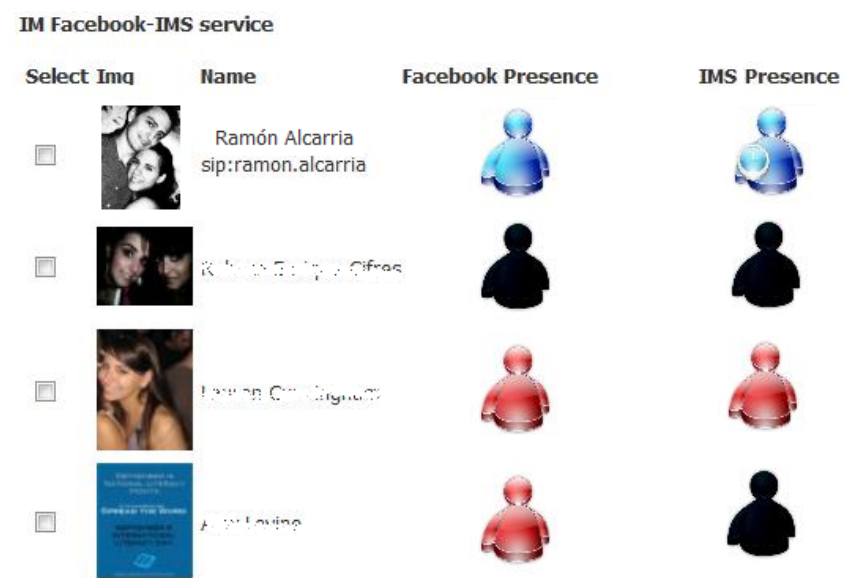

Figure 7. Proof of concept, part three. 
The integration possibilities between social and new generation networks are numerous, represent a very up-todate topic and are in continuous evolution. The vision of the integration by three levels (users, capabilities and services) permits new communication possibilities in messaging and multimedia information and also an improvement in the provided functionalities by the network to its users in the presence, group management and service provision fields.

\section{FUTURE WORK}

Future work in this field will be held in two ways. Following the first line, new interfaces for IMS access will be developed and we will improve the current interface with new functionalities, such as real time audio and video transmission. In addition, more proofs of concept will be carried out in order to validate the current work in IMS access. In these implementations we will keep special attention in developing integration mechanisms with other functionalities of social networks that we do not have taken into consideration in our current proof of concept, such as image sharing, service provision and group management.

\section{ACKNOWLEDGMENT}

This work has been carried out in the framework of the T2C2 project, whose objective is to investigate in the development of technologies which improve citizen cooperation to help coping with certain emergency situations by employing communication, computation and information resources provided by altruistic citizens.

\section{REFERENCES}

[1] N.D. Ziv and B. Mulloth, "An Exploration on Mobile Social Networking: Dodgeball as a Case in Point". International Conference on Mobile Business (ICMB), 2006.

[2] Y. Chang, H. Liu, and T. Wang, "Mobile social networks as quality of life technology for people with severe mental illness". IEEE Wireless Communications, Volume: 16, 2009.

[3] J. Rosenberg, H. Schulzrinne, G. Camarillo, A. Johnston, J. Peterson, R. Sparks, M. Handley, and E. Schooler, "SIP: Session Initiation Protocol," RFC 3261 (Proposed Standard), Jun. 2002. Available: http://www.ietf.org/rfc/rfc3261.txt

[4] 3GPP, "IP Multimedia Subsystem (IMS), Stage 2". 3rd Generation Partnership Project (3GPP), TS 23.228, Sep. 2008. Available: http://www.3gpp.org/ftp/Specs/html-info/23228.htm.

[5] T. O'Reilly, "What is Web 2.0? Design Patterns and Business Models for the Next Generation of Software", http://www.oreilly.com/pub/a/oreilly/tim/news/2005/09/30/what-isWeb-20.html, August 2005.

[6] D. Lozano, L. A. Galindo, and L. Garcia, "WIMS 2.0: Converging IMS and Web 2.0. Designing REST APIs for the Exposure of Session-Based IMS Capabilities". Next Generation Mobile Applications, Services and Technologies (NGMAST), 2008.

[7] O. J. Bertin, "Integrating IMS with web services to enable IP Multimedia Service Oriented Architectures". Internet Multimedia Services Architecture and Applications (IMSAA), 2008.

[8] T. Mecklin, M. Opsenica, H.-M. Rissanen, and D. Valderas, "ImsInnovation - experiences of an IMS testbed". Testbeds and
Research Infrastructures for the Development of Networks \& Communities and Workshops, 2009. TridentCom 2009.

[9] SIP Servlet API v1.1, Java Community Process Std. JSR 289, 2008.

[10] The ImsInnovation Developer Portal. IMS Rest API: https://labs.ericsson.com/apis/mobile-java-communicationframework/documentation/ims-rest-api

[11] Facebook Java Api, http://code.google.com/p/facebook-java-api/.

[12] P. Ekler and T. Lukovszki, "Similarity Distribution in PhonebookCentric Social Networks". 5th International Conference on Wireless and Mobile Communications (ICWMC 2009), 359-364, 2009.

[13] Nomadiclab: Ericsson Finland's research unit belonging to Ericsson's global research organization. http://www.ericsson.com/fi/ericsson/nomadiclab/ 